\title{
Towards Consistent Smart Grid Architecture Tool Support: From Use Cases to Visualization
}

\author{
Christian Neureiter and Dominik Engel \\ Josef Ressel Center for \\ Smart Grid Privacy, Security and Control, \\ Puch/Salzburg, Austria \\ Email: $\{$ christian.neureiter, dominik.engel $\} @$ en-trust.at
}

\author{
Jörn Trefke, Rafael Santodomingo, \\ Sebastian Rohjans and Mathias Uslar \\ OFFIS - Institute for Information Technology \\ Oldenburg, Germany \\ Email: $\{$ surname $\} @$ offis.de
}

\begin{abstract}
The complexity of today's power systems is continuously increasing due to the paradigm shift towards Smart Grids. This leads to a large number of use cases that need to be covered by holistic future energy systems of which power grids are just one subsystem. However, providing information about those use cases in a structured way in order to obtain a common basis for information exchange has often not been considered in the past. Covering this gap has led to the development of use case templates in the IEC and the Smart Grid Architecture Model in the EU Mandate M/490. Furthermore, regarding the usability of these two approaches, the SGAM Toolbox and the Use Case Management Repository have been developed as appropriate tool support. In this paper, a third component for visual analysis purposes is introduced. The overall goal of the approach proposed in this paper is to integrate these three components into a comprehensive toolchain that supports the whole process from use case development to visualization in compliance to the findings of EU Mandate M/490.
\end{abstract} $M D E$

Keywords-Interoperability, Standardization, Security, SGAM,

\section{INTRODUCTION}

One key challenge for the realization of Smart Grid systems is handling (large-scale) distributed systems [1]. In a first step this means to structure available and new information that have different levels of quality and detail. Closing this gap can be done by clearly defined processes and reference architectures.

On a more abstract level, interoperability has the same goal: Specifying the structure of systems and interfaces among them. Therefore, it is not surprising that the results from the EU Mandate M/490, which was originally focused on standardization, gain more and more momentum and are used far beyond their intended scopes (e.g., Enterprise Architecture [2]). In particular, the Smart Grid Architecture Model (SGAM) [3] is of high interest in this context. Furthermore, supporting tools such as the Use Case Management Repository (UCMR) [4] help to apply appropriate templates, e.g., from IEC PAS 62559.

Due to the complexity of Smart Grid systems and the tight connection between different subsystems, various stakeholders from different domains and disciplines are involved along the engineering process. Due to the nature of Smart Grid systems as typically distributed control systems the engineering task is characterized by iterative development steps and refinement. The failure to keep the contributions of all stakeholders synchronized is a frequent source of inconsistencies in a system architecture.
A broadly accepted method to tighten the cooperation between the different stakeholders and to handle the engineering complexity are model-centric approaches. These approaches, in contrast to document-based approaches, enable all stakeholders to contribute to the same database and hence assure a common knowledge and understanding. An approved concept for model-centric approaches is Model Driven Architecture (MDA) as introduced by the Object Management Group (OMG) [5].

The SGAM and appropriately surrounding processes, as well as use case management linked to the SGAM [6] as defined in the M/490 can be applied using such model-based approaches. However, these processes, as well as SGAM models themselves, are not properly formalized and are therefore not machine-executable. This is a crucial open gap in terms of applicability [7] even if different approaches exist that utilize the SGAM in their context [8]. One of the required actions to be taken in order to solve this problem is defining a suitable metamodel for the artifacts in this context [9]. Another one is to provide comprehensive tool support to allow for a simple application of the processes and models. For these reasons this paper proposes an integrated toolchain that allows for use case development, mapping to a reference architecture, and visualization.

The remainder of the paper is structured as follows: In Section II the SGAM is briefly introduced, whereas in Section III the integrated toolchain is explained in detail-the UCMR in Subsection III-A, the SGAM Toolbox in Subsection III-B, and the visualization component in Subsection III-C. The applicability of the toolchain is shown in Section IV based on a representative modeling example. Finally, Section V summarizes and concludes the paper.

\section{The SMART GRID ARChitecture Model}

The Smart Grid Architecture Model (SGAM) [3] has been developed in the course of the EU Mandate M/490 to European standardization organizations in order to support the Smart Grid deployment. It was developed as a part of the reference architecture framework during the work of the mandate. There, it is used to identify and characterize the power system domains, with their different zones, and the ICT systems which are relevant to this analysis with a special focus on interoperability/standardization. ICT systems must be described including the relevant technological components (such as storage, processing power and bandwidth) and the 
appropriate ICT objectives (such as resilience, maintenance, privacy and cyber security, and interoperability).

The SGAM defines a coordinated set of architecture viewpoints, informal concepts, as well as a method to map use case information to architectural artifacts and thus provides a structured approach for Smart Grid architecture development. Key to the SGAM are its five interrelated architecture viewpoints, addressing business, function, information, communication, and component concerns. These concerns can be traced back to the "Interoperability Framework" defined in [10] and are defined as interoperability layers in the SGAM. Each of these five layers further addresses organizational concerns and zones of power system management and relates them accordingly. Organizational concerns in this context are named "Domains" and defined according to the energy conversion chain/market roles. These include Generation, Transmission, Distribution, and Distributed Energy Resources (DER). Additionally, the domain Customer Premises comprises both, end users of electricity and producers of electricity, which include industrial, commercial, and home facilities as well as generation in the form of, e.g., photovoltaic, batteries, and micro turbines [3]. The zones of power system management include six hierarchical zones with decreasing distance to the physical network: Process, Field, Station, Operation, Enterprise, and Market.

Depicting the overall relationships of the interoperability layers, domains, and zones in the SGAM then provides a powerful tool to communicate about particular architectures on a high-level. For that, the SGAM defines a five-layered, cubelike visualization. However, the whole SGAM framework does neither provide any formal models to start with nor information on the concrete application in a project context yet.

However, tool support for the SGAM is crucial due to the complexity of its interrelated elements and development has already begun as can be seen in this paper. The extensions needed for toolchain integration enlarge the envisioned scope for the SGAM, making the methodology in conjunction with the IEC 62559 use case template easier to be put into daily utility practice, e.g. for IT/OT integration. This comprises "whatif" scenarios and using the SGAM model and scenarios for a Smart Grid maturity assessment, as envisioned for instance by the Software Engineering Institute at the Carnegie Mellon University and its Smart Grid Maturity Model SGMM, can be linked and make for easier business development. However, SGAM can also be used not only for future business models but also incorporate current grid codes and non-functional requirements into one single coherent model and modelling tool. The proposed toolchain and extensions will largely broaden the scope and acceptance of the UCMR and SGAM, but will also lead to a better toolbox for utilities for creating new business models as well as down to ground IT analysis functions like NISTIR-7628-based security analysis.

To sustainably apply the SGAM in development projects, it needs to be incorporated into a toolchain relying on standardized exchange formats. The SGAM can outgrow its original purpose, proving to be a methodology which can be utilized in different scenarios like IT/OT integration, innovative business models, security analysis, and architecture management.

\section{A Modeling TOOLCHAIN}

Different tools have been developed on the basis of work conducted in the wider context of the SGAM, in order to facilitate the operational use of the model itself. In detail, this section introduces three of them and integrates them into an overall toolchain as depicted in Fig. 1.

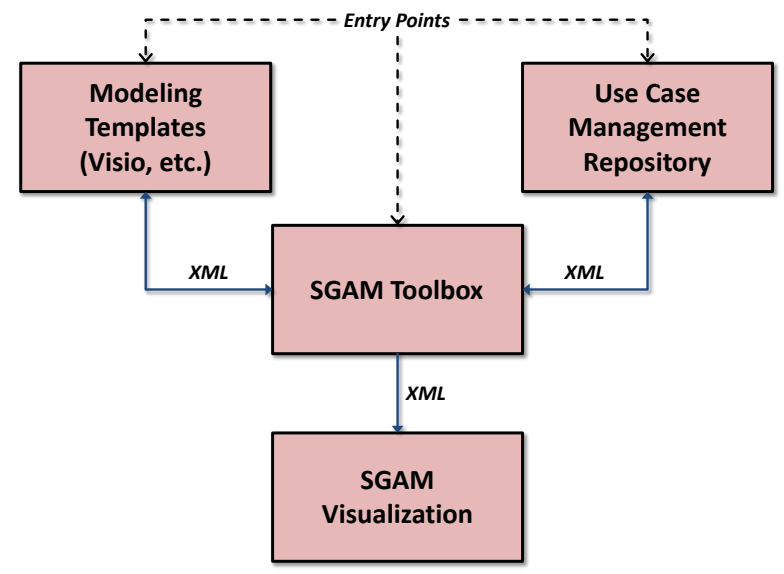

Fig. 1. Overview on the proposed toolchain

The principle idea is to reuse existing, widely accepted tools and link them in a toolchain in order to provide for a faster project success. Therefore, different entry points are provided. According to the situation of the potential user, the following entries are possible:

- Modeling Templates: In case only few and unstructured information are available, the user can start with simple SGAM templates, e.g., using Microsoft Visio or PowerPoint. A first system overview can be quickly modeled. During the next steps, these information can be imported by the SGAM Toolbox ${ }^{1}$ and then refined. Afterwards, the use case can be stored in the UCMR and/or be visualized.

- SGAM Toolbox: For requirements engineers and system architects the toolbox is the best entry point for re-engineering projects. Again, after modeling, the use cases can be stored in the UCMR or visualized.

- UCMR: This is the recommended entry point for developing new use cases. All information can be organized in a structured way from scratch. Afterwards, the SGAM Toolbox can import all use case information and allow for a mapping on the SGAM and further refinement. Finally, the SGAM mapping can be visualized.

Finally, the SGAM visualization component provides for visual analytic views. Different kinds of easily accessible visualizations will help the user to identify components, e.g., with highest OPEX or CAPEX costs, compare visually between different implementations of the very same functionality, and obtain an entry point for discussions with interdisciplinary experts.

\footnotetext{
${ }^{1}$ http://www.en-trust.at/SGAM-Toolbox
} 
The three components of the toolchain will be explained in the following subsections.

\section{A. The Use Case Management Repository}

Use-case-based requirements engineering for Smart Grids is gaining momentum as it enables the description of how a system behaves in relation to its stakeholders. This allows handling the complexity of systems and processes involved in Smart Grids. As more and more use cases for Smart Grids are developed, adequate management and coherent descriptions of use cases become necessary. The IEC Publicly Available Specification (PAS) 62559 [11] aims at providing a method to develop use cases for Smart Grids and provides a corresponding document template to gather relevant information. Further efforts in the IEC technical committee (TC) 8 working group 5 seek for a standardized template based on IEC PAS 62559 with supporting material for a computer-supported process. This includes, in addition to the template, also a process for gathering the data in a central repository as well as data exchange formats.

Besides the main purpose of collecting use-case-relatedinformation, the development of use cases is a collaborative task conducted by several project members. Since a large number of use cases are expected within complex Smart Grid projects this can easily lead to inconsistent information, redundancy, or unwanted editing conflicts when carried out in an uncontrolled process. Technically this editing process requires the multi user support, including roles and access control, locking, commenting, release, and configuration management of related artifacts. Along with a large number of use cases comes the need for classification, grouping, searching, and navigation of use cases that should be supported. Further, uniform semantics should be used, redundancies be avoided, and descriptions be complete. Consistency may in this case be achieved by using a comprehensive model and respective tool support. Additionally multiple languages could increase acceptance within international standardization and projects.

Ideally, the entire process of use case development, management, and its use for further development should be supported by an integrated tool, which allows interlinking information across multiple use cases keeping consistent semantics. To support the requirements stated above, the DISCERN project develops a Use Case Management Repository (UCMR) that implements the IEC 62559 template and also incorporates SGAM-related elements for further architecture definition. In order to structure and organize use cases, further measures are developed in the UC4AAL project, which can be applied in the context of Smart Grids:

- Conventions and guidelines: Conventions and guidelines ensure consistency throughout the development and management process.

- Template for use case development: The internal template for use case description contains every attribute without restriction to a specific user role. It is advisable to restrict the application of the general template to use case experts and administrators. For regular users, a role-specific template should be provided instead. This reduces the complexity and ensures conceptual clarity within use case development.
- Organization and classification of use cases: Rules for organization and classification of use cases, which improve accessibility and application, especially for large numbers of use cases.

The UCMR is implemented as a web-based application providing a "single-point of truth" for use case related information. Due to its web-based nature it enables collaborative, textbased editing of use cases among different experts within a company, or even from different companies. In addition to that, it performs analyses on the use case data to extract relevant information for users and to export it to other tools. Figure 2 shows the user interface for the UCMR in the editing dialog. The editing dialog shown in the central part enables users to

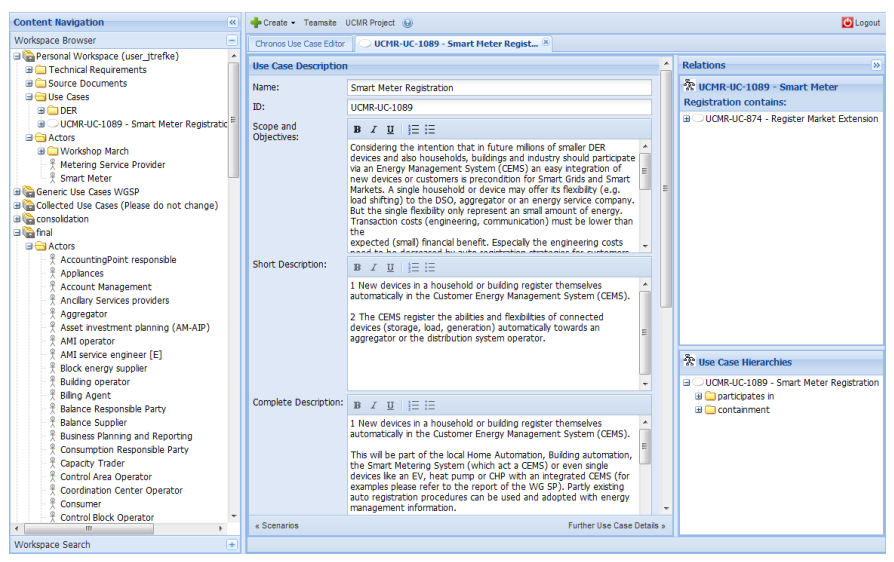

Fig. 2. Use Case Management Repository web-application, editing process

edit use cases and displays only relevant parts of the template according to the user's role. Users editing a use case can reuse existing elements from the repository by dragging them from the left-hand side and dropping them on respective input fields in the editing dialog. Furthermore relations to other use cases can be seen on the right hand side or new relations to other use cases be established.

\section{B. The SGAM Toolbox}

The SGAM Toolbox aims at supporting model driven engineering for Smart Grid systems. To be more precise, the concepts of Model Driven Architecture (MDA) should be introduced to engineering Smart Grid systems as discussed in [9]. Hereby a special focus is put on the early project phases "System Analysis" and "System Architecture" that typically require a close cooperation between domain experts and engineers. A crucial requirement for this task is the establishment of a common language between the different stakeholders. In technical terms this can be realized by the development of a Domain Specific Language (DSL) which can be used in the model driven engineering workflow. The SGAM, as described in Section II, more and more turns out to be a suitable basis for a common understanding of Smart Grid systems. Thus, the concepts of the SGAM are utilized for the development of a DSL that can serve as a modeling basis. A top-level overview of the DSL's architecture is shown in Fig. 3. The introduced DSL reflects the five layer architecture from the SGAM. Moreover, it shows the separation between the functional architecture (Business and 


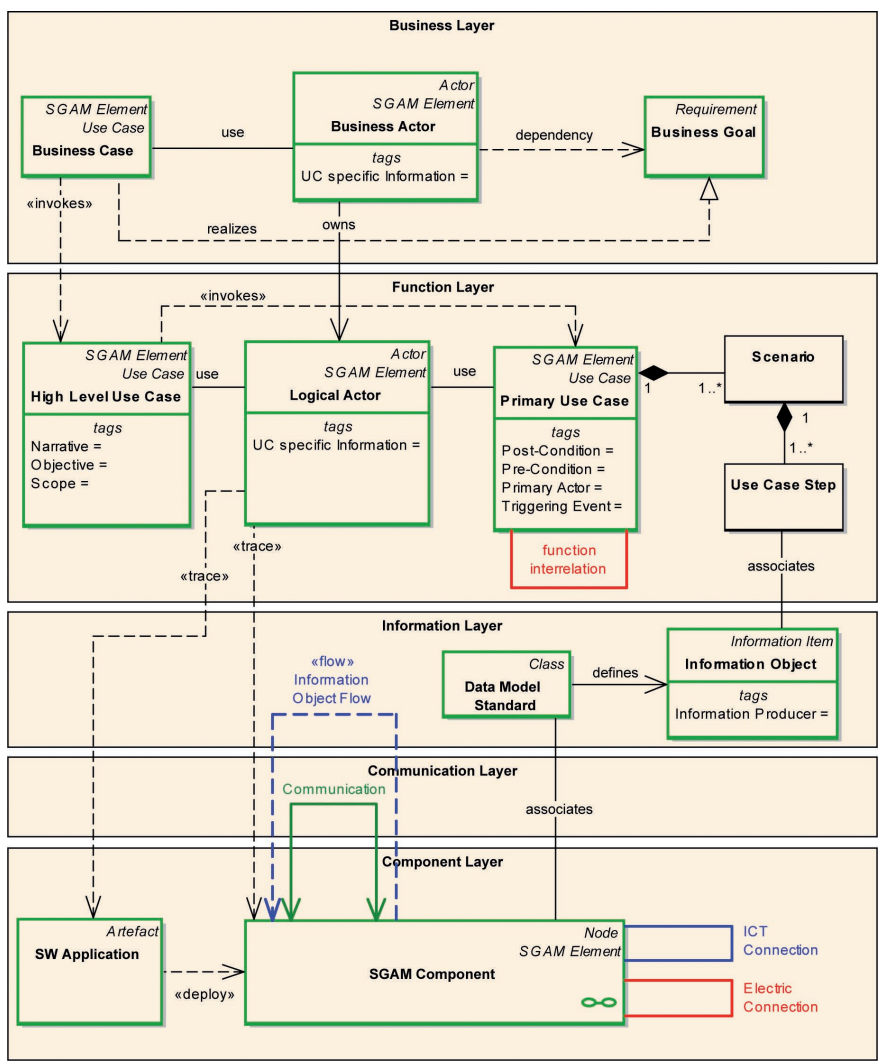

Fig. 3. Domain Specific Language model

Function layer) and the technical architecture (Information, Communication and Business layer). Thus, one of the basic requirements of MDA, the separation between functionality and technology is satisfied. The model transformation from functional elements, as for example elements of type "Logical Actor", into technical elements like "Components", is denoted by a "trace" relation.

One of the main concerns of model-centric approaches is the usage of different viewpoints to the same elements in order to address different aspects of a specific system. Thus, in the architectural view (lower three levels) the basic entity "SGAM Component", that is located in the Component layer, is reused in the Information and Communication layer to develop the information and communication specific aspects. In fact, the different SGAM layers represent-in terms of MDA-different viewpoints on a single, architectural solution for a preliminary described functionality (Business and Function layer).

In order to dispose of a broad tool support, the DSL as described above is implemented as extension to UML 2 by means of UML profiles. The implementation was done as extension to the "Enterprise Architect" 2 modeling tool by making use of the integrated Model Driven Generation functionality. Beyond that, numerous supporting elements like templates, reference examples and video tutorials have been created. All developed artifacts are publicly available as the aforementioned "SGAM Toolbox".

\footnotetext{
${ }^{2}$ http://www.sparxsystems.com
}

\section{SGAM Visualization Component}

The SGAM Visualization provides different views on SGAM-compliant architecture models for visual analysis, high-level navigation, and communication. Its core SGAM architecture view visualizes architecture data on the corresponding layer according the SGAM in the three-dimensional space. In addition to the visualization, the SGAM Visualization component allows limited interaction with these displayed information, e.g., rotation around the layers, zooming into the model, hiding of certain elements/layers or textual information of selected entities. As can be seen in the screenshot of the SGAM architecture view in Fig. 4, the application consists of a large canvas on the left side displaying the visualization and a control panel on the right. The interaction with the visualized

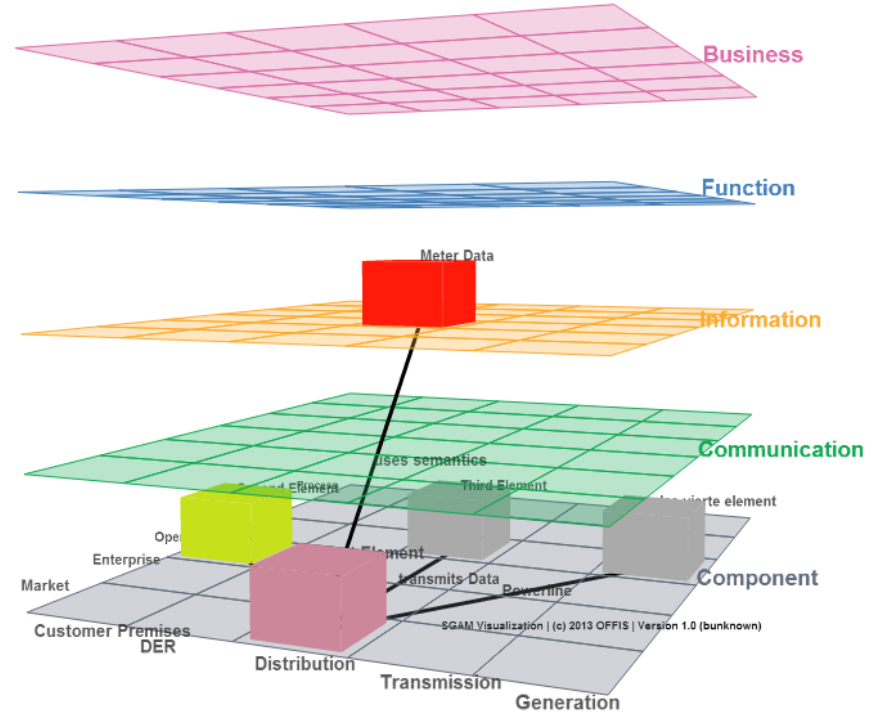

Fig. 4. SGAM Visualization component: architecture view

model can simply be controlled with the mouse. In addition to the architecture view a text-based view is available to explore the visualized data (not shown in the figure). The SGAM Visualization can import multiple architecture models and visualize them in one single view, i.e. models created with the SGAM Toolbox or parts thereof, and merge and visualize them accordingly, even if they were created by multiple authors. So it can contribute to a common understanding, the identification of shortcomings in a model, or to develop a holistic picture.

Technically, the visualization is realized as a mainly clientbased web-application that runs in most modern HTML 5 compatible web-browsers. The application can import basic XML files, which are transformed with XML-stylesheets and so can easily be extended to support various XML-based file formats (like UML models serialized in the XMI-format or formats used by common spreadsheet programs). In particular an Enterprise Architect/XMI-import is available to seemlessly integrate into the presented toolchain.

Beyond the features outlined above, future versions of the SGAM Visualization will include additional views and interactive analyses based on architectural properties, e.g., dependency analyses for selected architectural artifacts (e.g. for outages), architectural-property-based visualization (e.g., 
for safety-critical elements), as well as operations on certain architectural properties (e.g., aggregation of costs for certain elements).

\section{Modeling EXAMPLE}

To demonstrate the intended use of the integrated toolchain a simple example with a minimum of complexity is given. The example scenario assumes a distribution network with numerous fluctuating, decentralized electricity producers like photovoltaic systems and wind energy plants. In order to keep the voltage level stable, the Distribution System Operator (DSO) is interested in using shiftable loads to compensate the volatile behavior of the Distributed Energy Resources (DER). The given example assumes the presence of electric vehicle (EV) charging stations in the Distribution System that should be used for Demand Side Management (DSM).

Following the considerations from [9] in a first step, during the System Analysis phase, the given system context and the desired functionality are to be analyzed by the requirements engineer. In the example scenario, the DSO is the responsible party that is interested in the realization of the system in order to fulfill a specific business goal. The business goal of the DSO is to keep the electric parameters of the distribution system inside the preceptive limits without reinforcement of the electric system.

Once the required knowledge about the primary stakeholder (the $D S O$ ) and its specific goals has been obtained, additional stakeholders need to be identified in this phase. In the given example, the owner of the EV represents another stakeholder. The main interest of the EV owner, the User, is to have the EV fully loaded at a certain time. In addition to this primary goal, the user is interested in optimizing his or her energy costs. Hence, he or she is willing to shift the charging over time for a certain incentive, as long as the vehicle is fully charged at a previously defined time.

Being aware of the present stakeholder and their individual goals, the requirements engineer uses the use case templates as provided in IEC PAS 62559 to specify the logical actors, the realized use cases and the logical information items exchanged. Regarding the presented toolchain, the UCMR could be employed for this task as an entry point.

For the example scenario four logical actors can be identified: Two of them, the $D S O$ and the User are directly related to the preliminarily identified stakeholders, which are formally denoted as business actors. These two identified actors are complemented by the EV Charging Station and a central Energy Management System (EMS). The EMS realizes the main functionality, as it mediates between the interests of the DSO and the User and communicates the operation to be performed to the EV Charging Station.

The overall operation can be described as a High Level Use Case (HLUC) "Schedule E-Car Charging", which can be decomposed into three Primary Use Cases (PUC). The first PUC, titled "Preferences" involves the interactions between the User and the EMS. During the execution of this PUC the User communicates his charging preferences to the EMS. This preference could for instance be: Charge the EV when the energy tariff is low, but ensure it is fully loaded at a certain time.

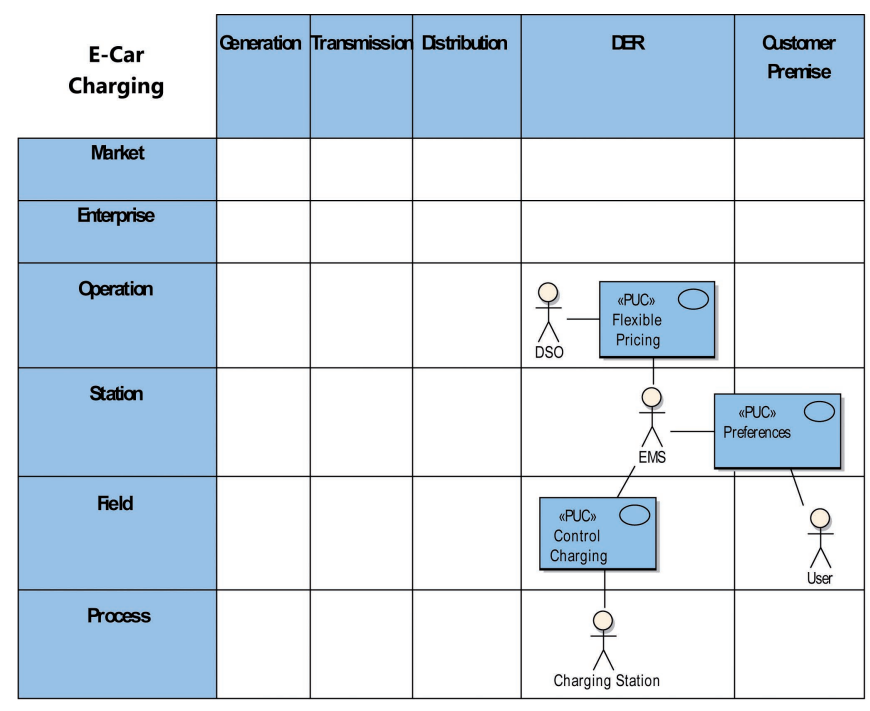

Fig. 5. SGAM function layer for the example scenario

The interactions between the DSO and the EMS are described in the PUC "Flexible Pricing", which comprises the communication of the actual energy tariff from the DSO to the EMS. In response to the described two PUCs that deliver the different interests of the DSO and the User to the EMS, the EMS is able to control the EV Charging Station. This PUC, titled "Control Charging", determines the optimal time for charging and controls the Charging Station in an appropriate manner.

After the functionality of the HLUC has been described within the use case template, the System Architecture phase can be executed. The responsible role in this phase is the system architect that turns the functional description into an architectural solution. For this task, the SGAM Toolbox can be used. The SGAM Toolbox comprises functionality for importing the information from the Use Case Template into a model utilizing the DSL as described in Section III-B. To be more precise, the Business and the Function layer of the SGAM can be synthesized based on the information available in the use case template. In terms of MDA these two layers represent the Computation Independent Model (CIM). Figure 5 depicts the synthesized Function layer for the discussed demonstration example.

Subsequent to this, a model transformation from the CIM to the Platform Independent Model (PIM) can be done. This means, the logical actors are mapped to physical components. These components and their electric and ICT relations are depicted in the Component layer of the SGAM, which is shown in Fig. 7. The actor mapping model representing the model transformation is illustrated in Fig. 6. In the given example, the logical actors are mapped to the physical components Distribution Management System (DMS), Energy Management System (EMS), Charging Station and Charging UI.

With the Component layer having been developed, further architectural development concerning the communication architecture and the information models can be done by creating and elaborating the Information and the Communication layer. When the architecture is modeled in this way, numerous further 

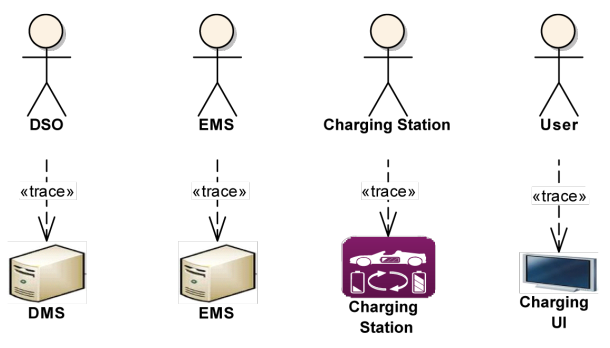

Fig. 6. Actor mapping model for CIM to PIM transformation

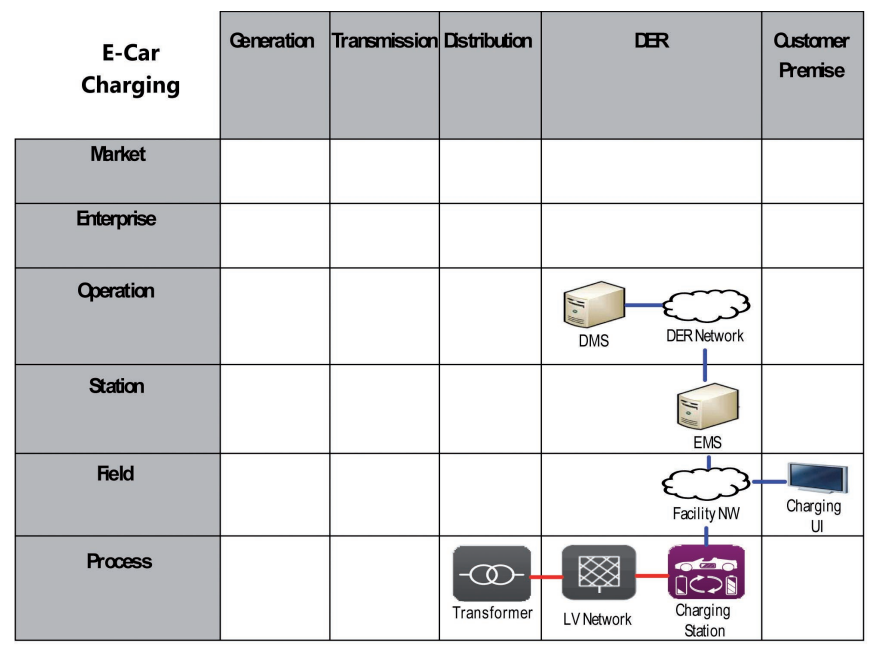

Fig. 7. Component layer for the example scenario

proceedings are possible. Besides various analytic assessments as supported by the SGAM Toolbox, the developed architecture can be exported to the UCMR to be reused as starting point for future projects. In addition, the model can be exported to the SGAM Visualization for further analytics. The visualization then provides an overview over all architectural elements defined, which can be explored visually. Moreover, further architecture models realizing another use case can be imported and interrelations between them can be identified.

\section{Summary ANd CONClusions}

In order to cope with the increasing complexity in future energy systems in terms of an increasing number of use cases, an appropriate tool support is needed. First, existing and new information have to be structured, then they have to be brought in line with an accepted reference architecture, and finally they have to be suitably visualized. For satisfying these requirements existing tools need to be integrated into a comprehensive toolchain. For this purpose, the UCMR (developing use cases), the SGAM Toolbox (modeling use cases based on an established reference architecture), and a visualization component (providing an entry point for discussion based on a common understanding) have been introduced. It has been shown that by a combination of these three components, comprehensive tool support can be achieved.

One key issue addressed by future work is the specification of the exchanged XML files. Aiming at an increased interoperability for the exchange of SGAM-based models, the appropriate exchange formats have to be standardized (as can be seen for the IEC 62559 template) or at least be published as an international technical report. This implies to provide the specification to the responsible IEC working groups. For this process, the Common Information Model (CIM; IEC 61970/62968) and IEC 61850 give good examples how XMLbased information exchange can be realized and standardized. Finally, all three components are continuously improved and developed.

\section{ACKNOWLEDGMENT}

The research leading to these results has received funding from the European Union Seventh Framework Programme (FP7/2007-2013) under Grant Agreement no 308913, "DISCERN".

The research leading to these results has received funding from the German Federal Ministry for Economic Affairs and Energy under Grant Agreement no 01FS13028, "UC4AAL".

The financial support of the Josef Ressel Center by the Austrian Federal Ministry of Economy, Family and Youth and the Austrian National Foundation for Research, Technology and Development is gratefully acknowledged.

Funding by the Austrian Federal Ministry for Transport, Innovation and Technology and the Austrian Research Promotion Agency (FFG) under Project 838793, "INTEGRA", is gratefully acknowledged.

\section{REFERENCES}

[1] X. Fang, S. Misra, G. Xue, and D. Yang, "Smart Grid - The New and Improved Power Grid: A Survey," IEEE Communications Surveys \& Tutorials, vol. 14, no. 4, pp. 944-980, 2011.

[2] J. Trefke and C. Dänekas, "Development of Smart Grid Architectures," in Standardization in Smart Grids, ser. Power Systems. Springer Berlin Heidelberg, 2013, pp. 59-77.

[3] Smart Grid Coordination Group, "Smart Grid Reference Architecture," CEN-CENELEC-ETSI, Tech. Rep., 2012.

[4] J. Trefke, J. M. González, and M. Uslar, "Smart Grid Standardisation Management with Use Cases," in 2nd IEEE ENERGYCON Conference \& Exhibition, Florence, Italy, 2012, pp. 966-971.

[5] Object and Reference Model Architecture Board Subcommittee, "Model Driven Architecture (MDA)," Object Management Group, Tech. Rep., 2001.

[6] Smart Grid Coordination Group, "Sustainable Processes," CENCENELEC-ETSI, Tech. Rep., 2012.

[7] F. Andren, T. Strasser, S. Rohjans, and M. Uslar, "Analyzing the Need for a Common Modeling Language for Smart Grid Applications," in 11th IEEE International Conference on Industrial Informatics, 2013.

[8] J. Trefke, S. Rohjans, M. Uslar, S. Lehnhoff, L. Nordström, and A. Saleem, "Smart Grid Architecture Model Use Case Management in a large European Smart Grid Project," in 4th IEEE European Innovative Smart Grid Technologies (ISGT), 2013.

[9] C. Dänekas, C. Neureiter, S. Rohjans, M. Uslar, and D. Engel, "Towards a model-driven-architecture process for smart grid projects," in Digital Enterprise Design \& Management, ser. Advances in Intelligent Systems and Computing, P. Benghozi, D. Krob, A. Lonjon, and H. Panetto, Eds. Springer International Publishing, 2014, vol. 261, pp. 47-58.

[10] The GridWise Architecture Council, "GridWise Interoperability Context- Setting Framework," Tech. Rep., 2008. [Online]. Available: http://www.gridwiseac.org/pdfs/interopframework $\backslash$ _v1 $\backslash \_1 . p d f$

[11] International Electrotechnical Commission (IEC), "Publicly Available Specification (PAS) 62559 IntelliGrid Methodology for Developing Requirements for Energy Systems," Tech. Rep., 2008. 